\title{
Response-dependent effects on near-threshold detection performance: Saccades versus manual responses
}

\author{
HOWARD C. HUGHES and JAMES V. KELSEY \\ Dartmouth College, Hanover, New Hampshire
}

\begin{abstract}
The inverse relationship between flash intensity and response times was studied under identical conditions using two different response measures, saccadic eye movements and manual responses. It was found that saccade latencies were less dependent on flash intensity than were manual responses. In addition, detection failures to near-threshold flashes were significantly less frequent when subjects signaled detection of the flash by making a saccade. These differences in detection performance may reflect a greater capacity of the oculomotor system to decode near-threshold ganglion cell responses.
\end{abstract}

It is well known that reaction times (RTs) are inversely related to signal intensity. The question addressed by the present experiment is: Do the details of this relationship depend in any way on the nature of the detection response? If so, such differences would presumably reflect important differences in either the sensitivity or the detection criteria employed by the visuomotor pathways that mediate the detection responses. Wheeless, Cohen, and Boynton (1967) reported that saccade latencies to target displacements away from the fovea varied by approximately $60 \mathrm{msec}$ over an intensity range extending from the foveal threshold to $2 \log$ units above threshold. In contrast, the intensity-dependent variation in manual reaction times can vary by twice this much, even at luminances well above threshold (e.g., Mansfield, 1973). Although it thus seems possible that saccade latencies might be less dependent on target luminance than manual response times, this possibility has not been specifically evaluated. The present experiment addressed this question directly, by comparing saccadic and manual reaction times under identical conditions in the same subjects. The results indicate that saccades are indeed less dependent on luminance than are manual responses, both in terms of detection latency and the frequency of detection failures, indicating that important aspects of sensory performance are partially response-dependent.

\section{METHOD}

\section{Response Recording}

Horizontal eye position was recorded via bitemporally placed dc recording electrodes. The signals were digitized (sampling rate of

This research was supported in part by a fellowship awarded by the Alfred P. Sloan Foundation, and in part by a faculty fellowship awarded by Dartmouth College. J.V.K. was supported by funds supplied by Dartmouth College. The authors' mailing address is: Department of Psychology, Dartmouth College, Hanover, NH 03755.
$500 \mathrm{~Hz}$ ) following high-gain differential amplification. The signalto-noise ratio of these recordings permitted resolution of eye movements greater than $1 \mathrm{deg}$ in amplitude (see Figure 1).

Subjects responded manually by depressing a microswitch with the index finger of the right hand. The microswitch was polled every millisecond by a clock-driven interrupt routine.

\section{Stimulus Display}

The stimulus display consists of an array of nine light-emitting diodes (LEDs) aligned at 10-deg intervals along the horizontal meridian. Only two of these were used in the present experiment, however-a centrally located fixation light and a target light located $10 \mathrm{deg}$ to the right of fixation. The LEDs are mounted in a white Plexiglas panel that is $10 \mathrm{~cm}$ wide bent in an arc of $100 \mathrm{deg}$. The panel was diffusely illuminated by a dc halogen bulb that provided a background illumination of $.77 \mathrm{kx}$. The LEDs were illuminated intermittently at a temporal frequency of $100 \mathrm{~Hz}$ with a minimum pulse width of $10 \mu \mathrm{sec}$. Intensity was controlled by varying the pulse width from 10 to $9,999 \mu \mathrm{sec}$, which produced a linear increase in the radiometrically measured output from .02 to $20 \mu \mathrm{W} / \mathrm{cm}^{2}$.

The observers viewed this display binocularly at a distance of $57 \mathrm{~cm}$. Head movements were minimized by using a chinrest.

\section{Observers}

Six observers served as subjects. Each had received at least 100 practice trials on both responses prior to the start of the experiment. All subjects claimed to be emmetropic or were appropriately corrected.

\section{Procedure}

Reaction times were obtained in all six subjects (without feedback), using both response measures at four different flash intensities (1.25, 2.0, 10.0, and 100.0 times the subject's target detection threshold). During preliminary sessions, thresholds were determined using the method of adjustment. The duration of the target flash was $10 \mathrm{msec}$. The flash always occurred $10 \mathrm{deg}$ to the right of fixation following the presentation of a warning tone $(1000 \mathrm{~Hz}, 100$-msec duration). A rectangular distribution of foreperiods ranging from 500 to $1,500 \mathrm{msec}$ was used. Trials were initiated by the subject. Eye position was monitored from the beginning of a trial until $1 \mathrm{sec}$ after the flash. Deviations from fixation during the trial caused that trial to start over. RTs less than $100 \mathrm{msec}$ were considered anticipatory and were not included in the formal analysis. Such anticipations were very rare, however, as indicated in the latency histograms of Figure 4. The four flash intensities were randomized within blocks of 24 trials, and cach in- 


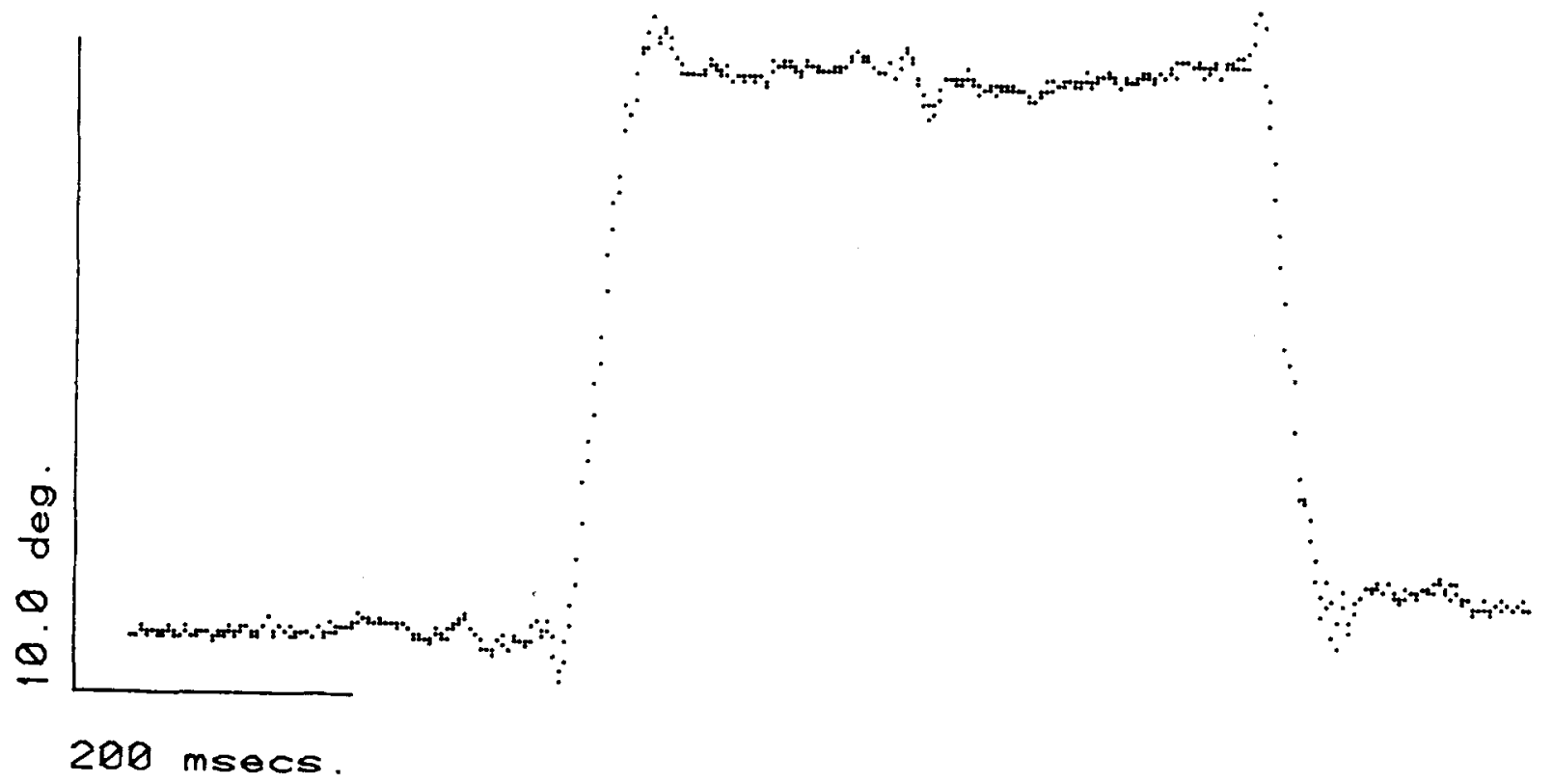

Figure 1. A record of a saccade typical of those recorded in the present experiments.

tensity appeared with equal frequency. All aspects of the experiment were under the control of a laboratory microprocessor.

Data were obtained over five daily experimental sessions. Each session consisted of $\mathbf{4 8}$ saccade trials and $\mathbf{4 8}$ manual trials, the order being counterbalanced across days. Thus, the total data sample from each subject consists of 60 reactions at each intensity for both types of response.

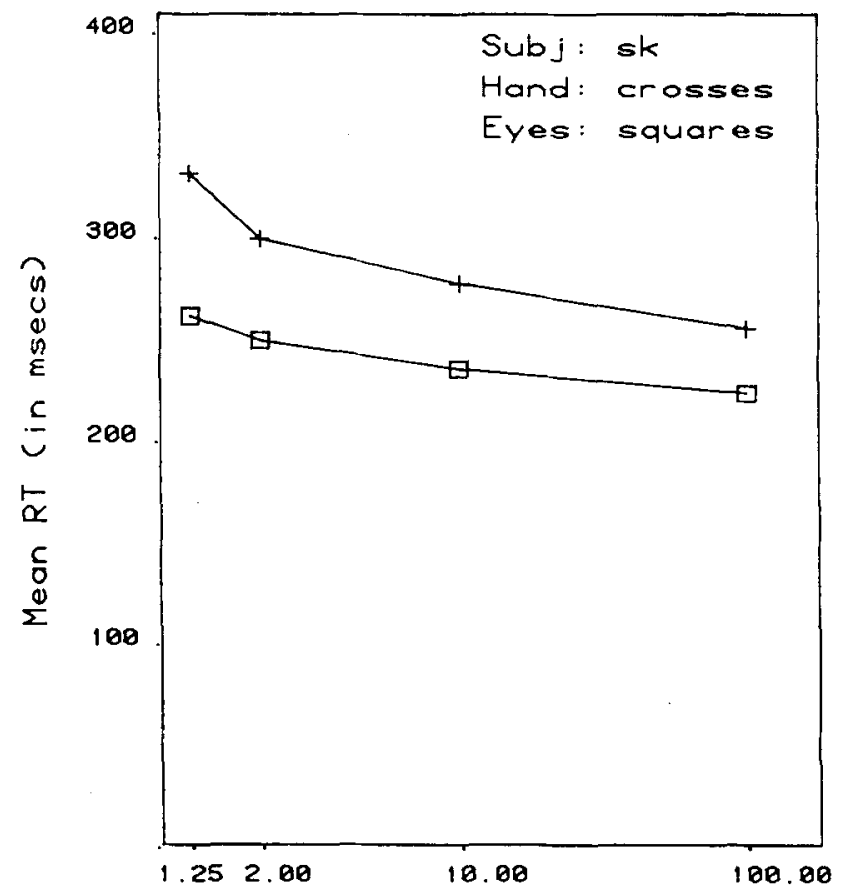

RESULTS

Figure 2 illustrates the intensity-RT curves for manual responses and saccades in two of the observers. In comparing the asymptotic latencies for both responses, it became clear that the asymptotes

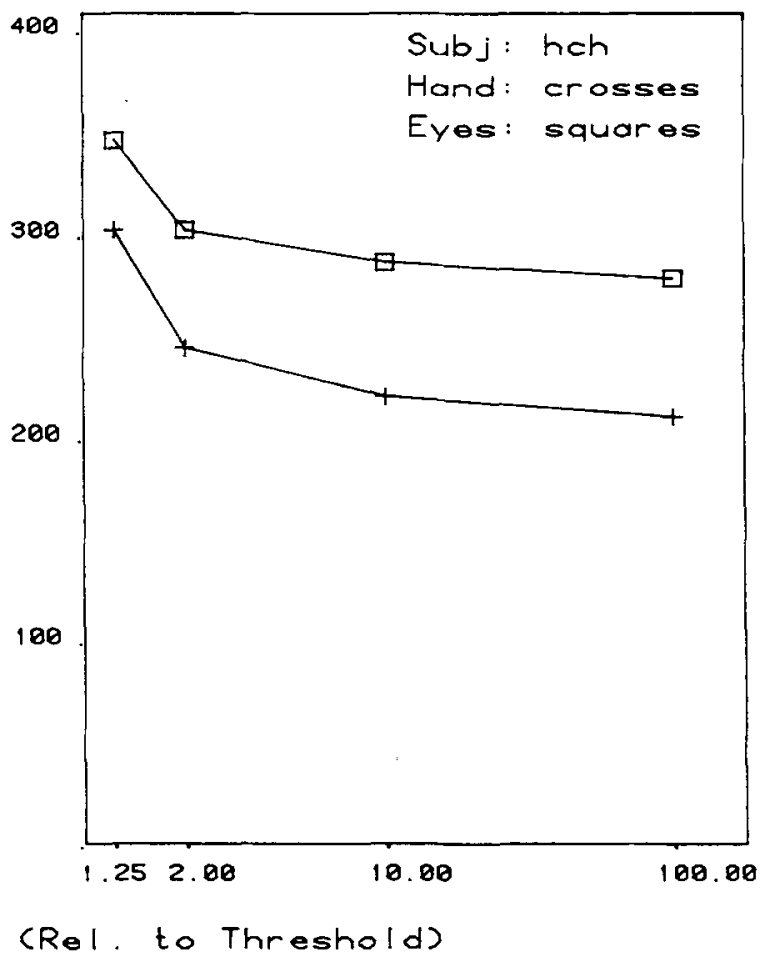

Figure 2. Intensity-reaction time curves for two observers. Saccade latencies are represented by the squares; manual latencies are represented by the crosses. 

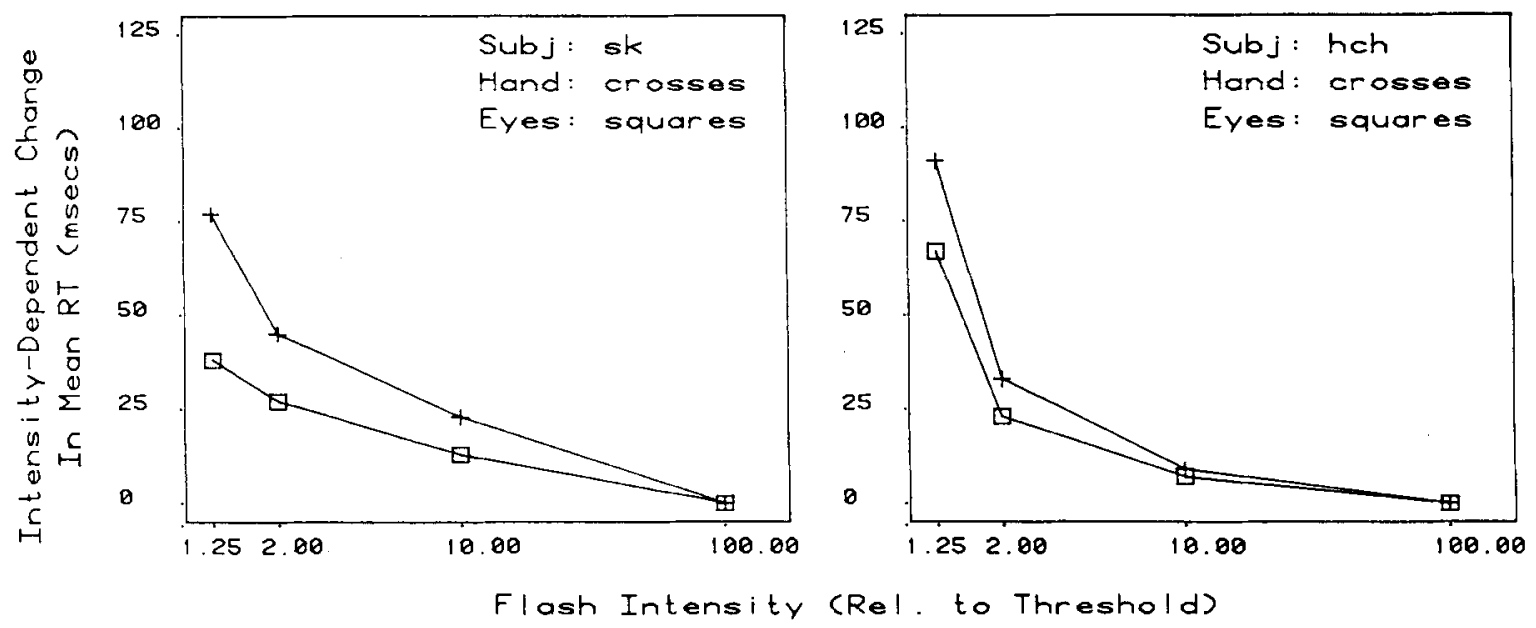

Figure 3. Intensity-dependent variation in simple reaction time for the same two observers as shown in Figure 2.

were subject to fairly substantial individual differences. In some subjects, saccades were faster than manual responses, whereas in others the reverse was true. In still others, the asymptotes were virtually identical. As in other studies of intensity-dependent effects on RT (e.g., Mansfield, 1973), these individual differences in asymptotic latency were removed by subtracting the mean latency for the bright flash from the times obtained using the other intensities. The resulting curves reflect only the intensity-dependent portion of each subject's curve, and examples are illustrated in Figure 3. The intensity-dependent means for each subject were analyzed via a threefactor analysis of variance (subjects $x$ intensity $x$ re-

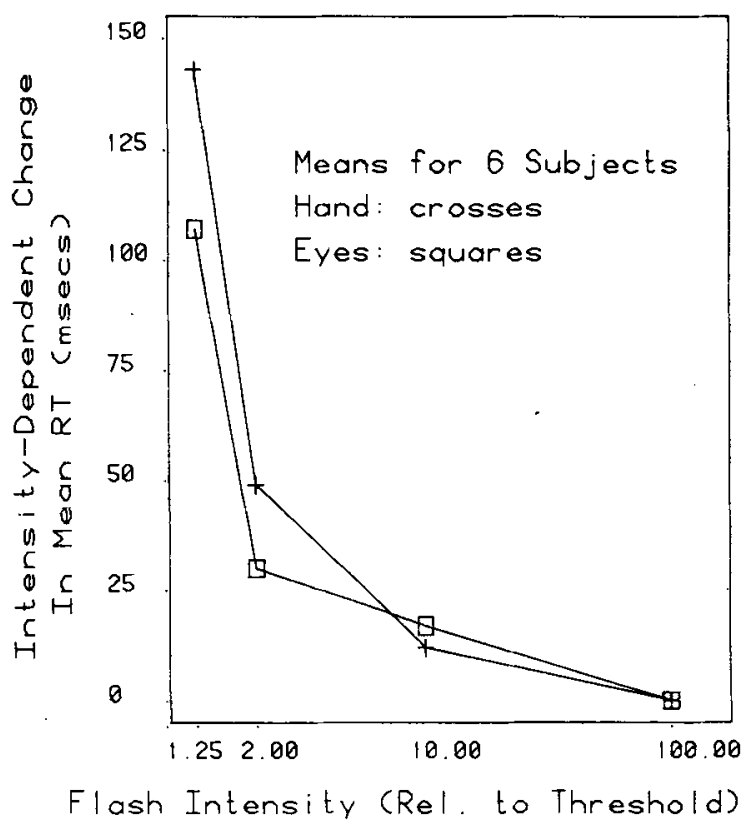

Figure 4. Intensity-dependent variation in saccade and manual latencies. The data points represent the means for all six observers.

$$
\text { Subj : JC }
$$$$
\text { Resp: Hand }
$$$$
\text { Resp: Eye }
$$$$
\text { Flosh Intensity }
$$
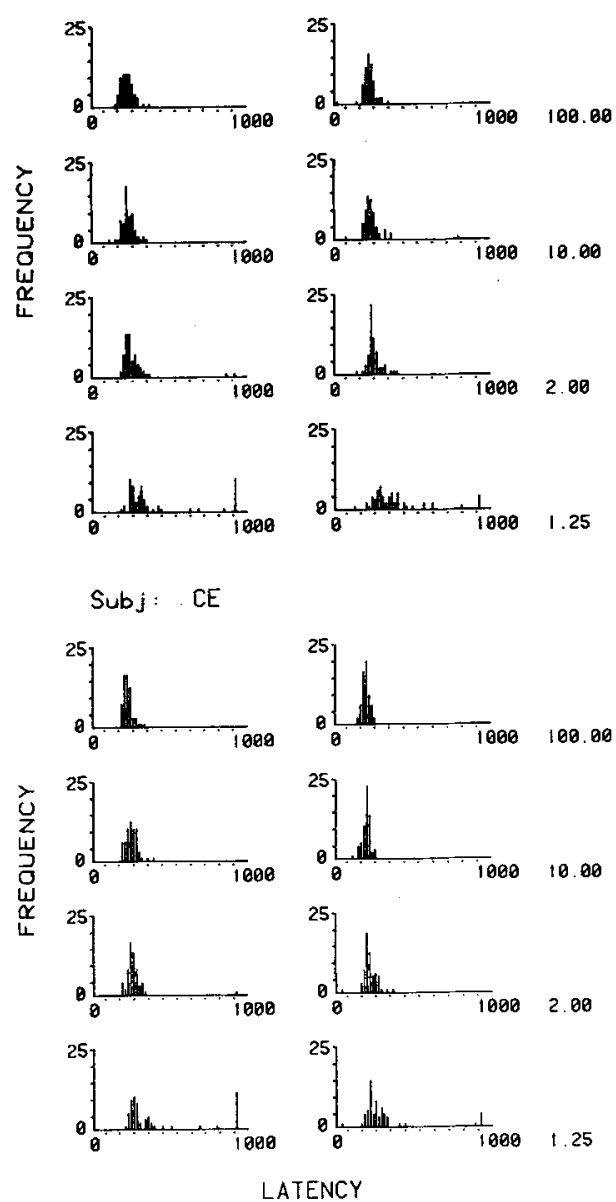

Figure 5. Latency histograms for saccades and manual responses in two observers. Manual reaction times are illustrated on the left, saccades on the right. Notice that, in both subjects, the frequency of detection failures (no response within $1,000 \mathrm{msec}$ ) was greater for manual responses than for saccades. 
sponse type), which indicated a significant main effect of intensity $[F(2,10)=26.3, p<.001]$ and a significant interaction between intensity and response type $[F(2,10)=4.6, p<.04]$. The main effect of response type was not significant. The averaged data for all six subjects are illustrated in Figure 4.

The interaction between intensity and response type is a major finding of the experiment, and results from the divergence of the intensity-RT curves with decreasing luminance (Figure 4). A second important finding is that the frequency of detection failures (defined by no response within the 1,000-msec postflash sampling invertal) was greater for manual responses than for saccades. This effect is illustrated in the histograms in Figure 5. When saccades were used as the detection responses, the subjects averaged 8.5 $(14.2 \%)$ misses to the dimmest flash, whereas they averaged $13.5(22.5 \%)$ misses with manual detection responses. An analysis of variance indicated that this difference was statistically reliable $[F(1,5)=8.33$, $\mathrm{p}<.04]$. Inspection of Figure 5 indicates that detection responses, when they occur, precede the 1,000 msec deadline by a substantial amount, supporting the interpretation that the duration of the sampling interval was sufficient, and that those trials in which a detection response was not emitted within 1,000 msec represent true misses rather than an artifact produced by long-latency responses that exceed the sampling interval. Thus, it would appear that the probability of detecting near-threshold targets is higher for the saccade system than for the manual system.

\section{DISCUSSION}

The results of this experiment showed that, under the same conditions of stimulation, saccade latencies are less dependent on target luminance than are man- ual responses. Since the same retinal position was stimulated on both manual and saccade trials, the source of this interaction presumably lies in differences in intensity-dependent delays of the central pathways underlying visually triggered eye and hand movements. Thus, while a substantial proportion of the intensity-dependent delays in visual processing are common to both saccade and manual RTs, there is also a significant residual contribution that cannot be attributable to photoreceptor response latency (Mansfield, 1973; Mansfield \& Daugman, 1980).

Although extrapolation of these latency effects to threshold luminances would imply a response-dependent difference in detection thresholds, we were surprised to see the indications that these differences may actually exist. In the domain of signal detection theory, there are two possible sources for the observed differences in detection rates for saccades and manual responses: a difference in sensitivity or a difference in response criterion (Green \& Swets, 1966). Although the present data do not permit a selection between these alternatives, future work will attempt to identify the source of this difference in these terms.

\section{REFERENCES}

Green, D. M., \& Swets, J. (1966). Signal detection theory and psychophysics. New York: Wiley.

MANSFIELD, R. J. W. (1973). Latency functions in human vision. Vision Research, 13, 2219-2234.

Mansfield, R. J. W., \& Daugman, J. A. (1980). Retinal mechanisms of visual latency. Vision Research, 18, 1247-1260.

Wheeless, L. L., Cohen, G. H., \& Boynton, R. M. (1967). Luminance as a parameter of the eye-movement control system. Journal of the Optical Society of America, 57, 394-400.

(Manuscript received November 14, 1983; revision accepted for publication April 16, 1984.) 